\title{
Factors Associated with Use of Inpatient Telehealth for Hospitalized COVID-19 Patients: an Observational Study of 4 Hospitals in a Single Health System
}

J Gen Intern Med 37(5):1325-7

DOI: $10.1007 / \mathrm{s} 11606-021-07220-7$

(c) Society of General Internal Medicine 2022

\section{INTRODUCTION}

Recent ambulatory studies have found differences in telehealth use based on race and ethnicity during the COVID-19 pandemic. $^{1-3}$ Whether racial and ethnic disparities exist in telehealth use in hospitalized patients is not clear. We examine variation in inpatient telehealth use for hospitalized COVID-19 patients based on demographic and clinical characteristics.

\section{METHODS}

We defined telehealth as a virtual visit using telephonic communication without a clinician physical examination. We included hospitalized COVID-19 positive patients with length of stay $\leq 30$ days from four hospitals in an integrated health system in Chicago, IL, from March-May, 2020. The study period ended in May 2020 due to an internal policy change in June requiring daily clinician physical exams of all patients. Our study sites included three mid-sized community hospitals (referred to as sites A, B, C; 159-390 beds) and one large academic medical center (site D; 894 beds).

All sites utilized the same electronic medical record and had consistent access to personal protective equipment. There was similar access to telehealth technology and limited use of residents. Telehealth visits were identified using an inpatient billing modifier code. A manual review of thirty medical records from each site validated the use of the modifier code with $>90 \%$ accuracy.

We conducted unadjusted analyses comparing demographic and clinical characteristics for patients with no telehealth visits to those with $\geq 1$ telehealth visit. We created multiple Poisson regression models, controlling for demographic, site of care, and clinical covariates and using length of stay as the exposure variable to identify characteristics independently associated with telehealth visits.

Received May 7, 2021

Accepted October 15, 2021

Published online February 7, 2022

\section{RESULTS}

Overall, 2,200 COVID-19 patients were evaluated, of which 1,142 patients $(51.9 \%)$ had $\geq 1$ telehealth visit during hospitalization with a median (IQR) of $3.0(1.0-5.0)$ among those with telehealth visits. Table 1 provides patient characteristics and unadjusted analyses. Using multiple Poisson regression, we found telehealth visit use was significantly associated with patient race, ethnicity, admission source, payer, month of admission, and site of care (Table 2). Race categorized as other was associated with less telehealth use compared to white race. Latinx ethnicity was associated with higher telehealth use. Site of care was strongly associated with telehealth use, with patients at one community hospital (site C) experiencing twice as many telehealth visits and patients at two other community hospitals (sites A and B) experiencing less than a third as many compared to the academic hospital (site D).

\section{DISCUSSION}

In this study of hospitalized COVID-19 patients, we found the rate of telehealth visit use was significantly associated with patient race, ethnicity, admission source, payer, month of admission, and site of care. Clinicians' decisions to conduct inpatient telehealth visits in place of in-person visits may have been influenced by various factors. Clinicians may have a desire to conduct rigorous in-person evaluations of patients with high acuity of illness or comorbidities. However, patients with higher acuity of illness may be perceived as higher risk for transmitting COVID-19 to clinicians. These countervailing forces may explain why we found no differences in telehealth use based on intensive care unit stay, mechanical ventilation, or Elixhauser score.

Though small, we found significant differences in telehealth usage based on race and ethnicity with a higher incidence in Latinx patients and lower use in other patients. Ambulatory studies have also found differences in telehealth use during the COVID-19 pandemic based on race and ethnicity. ${ }^{1-3}$ Access to technology explains some of the differences seen in the outpatient setting but is not applicable in our study as access to the technology needed to conduct telehealth visits was universal. 
Table 1 Characteristics of Hospitalized COVID-19 Patients by Occurrence of Telehealth Visit(s) in 2020

\begin{tabular}{|c|c|c|c|c|}
\hline & $\begin{array}{l}\text { No } \\
\text { televisits } \\
N=\mathbf{1 , 0 5 8}\end{array}$ & $\begin{array}{l}\geq 1 \\
\text { televisit } \\
N=1,142\end{array}$ & $\begin{array}{l}\text { All } \\
\text { patients* } \\
N=2,200\end{array}$ & $\overline{P \text { value }}$ \\
\hline Mean age (SD) & $55.8(18.5)$ & $56.6(17.2)$ & $56.2(17.9)$ & 0.31 \\
\hline \multicolumn{5}{|l|}{ Gender, $n(\%)$} \\
\hline Female & $506(47.8)$ & $554(48.6)$ & $1,060(48.2)$ & \\
\hline Male & $552(52.2)$ & 587 (51.5) & $1,139(51.8)$ & \\
\hline \multicolumn{4}{|l|}{ Race, $n(\%)$} & $<0.01$ \\
\hline White & $650(62.9)$ & $590(53.0)$ & $1,240(57.8)$ & \\
\hline Black & $188(18.2)$ & 329 (29.6) & $517(24.1)$ & \\
\hline Asian & $40(3.9)$ & $33(3.0)$ & $73(3.4)$ & \\
\hline Other & $156(15.1)$ & $161(14.5)$ & 317 (14.8) & \\
\hline \multicolumn{5}{|l|}{ Ethnicity } \\
\hline Latinx & $385(37.5)$ & $432(38.7)$ & $817(38.1)$ & \\
\hline Non-Latinx & $642(62.5)$ & $684(61.3)$ & $1,326(61.9)$ & \\
\hline \multirow{2}{*}{\multicolumn{5}{|c|}{$\begin{array}{l}\text { Primary } \\
\text { language }\end{array}$}} \\
\hline & & & & \\
\hline English & 801 (75.7) & $842(73.7)$ & $1,643(74.7)$ & \\
\hline Non-English & $257(24.3)$ & $300(26.3)$ & $557(25.3)$ & \\
\hline Mean BMI (SD) & $30.8(7.3)$ & $31.0(8.6)$ & $30.9(8.0)$ & 0.48 \\
\hline \multirow{2}{*}{\multicolumn{5}{|c|}{$\begin{array}{l}\text { Admission } \\
\text { source, } n(\%)\end{array}$}} \\
\hline & & & & \\
\hline $\begin{array}{l}\text { Emergency } \\
\text { department }\end{array}$ & $964(91.1)$ & $\begin{array}{l}1,050 \\
(91.9)\end{array}$ & 2,014 (91.6) & \\
\hline Direct & $75(7.1)$ & $64(5.6)$ & $139(6.3)$ & \\
\hline \multicolumn{5}{|l|}{ Admission } \\
\hline $\begin{array}{l}\text { External } \\
\text { transfer }\end{array}$ & $19(1.8)$ & $28(2.5)$ & $47(2.1)$ & \\
\hline \multicolumn{5}{|l|}{$\begin{array}{l}\text { transter } \\
\text { Payer, } n(\%)\end{array}$} \\
\hline Medicare & $449(42.4)$ & $494(43.3)$ & 943 (42.9) & \\
\hline Private & $383(36.2)$ & $386(33.8)$ & $769(35.0)$ & \\
\hline Medicaid & 179 (16.9) & $233(20.4)$ & 412 (18.7) & \\
\hline $\begin{array}{l}\text { Self-pay/ } \\
\text { other }\end{array}$ & $47(4.4)$ & $29(2.5)$ & $76(3.5)$ & \\
\hline $\begin{array}{l}\text { Intensive care } \\
\text { unit stav, } n(\%)\end{array}$ & $141(13.3)$ & $271(23.7)$ & 412 (18.7) & $<0.01$ \\
\hline $\begin{array}{l}\text { Mechanical } \\
\text { ventilation, } n(\%)\end{array}$ & $76(7.2)$ & 159 (13.9) & 235 (10.7) & $<0.01$ \\
\hline Elixhauser & $8.0(9.9)$ & $9.9(10.4)$ & $9.0(10.2)$ & $<0.01$ \\
\hline \multicolumn{5}{|l|}{$\begin{array}{l}\text { score, mean } \\
\text { (SD) }\end{array}$} \\
\hline \multicolumn{5}{|l|}{ Month, $n(\%)$} \\
\hline March & $139(13.1)$ & $166(15.4)$ & 305 (13.9) & \\
\hline April & 419 (39.6) & $594(52.0)$ & $1,013(46.1)$ & \\
\hline May & $500(47.4)$ & $382(33.5)$ & $882(40.1)$ & \\
\hline \multicolumn{4}{|l|}{ Hospital, n (\%) } & $<0.01$ \\
\hline A & 454 (42.9) & $98(8.6)$ & $552(25.1)$ & \\
\hline $\mathrm{B}$ & 123 (11.6) & $54(4.7)$ & $177(8.1)$ & \\
\hline $\mathrm{C}$ & $128(12.1)$ & $361(31.6)$ & 489 (22.2) & \\
\hline $\mathrm{D}$ & $353(33.4)$ & $629(55.1)$ & $982(44.6)$ & \\
\hline $\begin{array}{l}\text { Number of } \\
\text { televisits, } \\
\text { median (IOR) }\end{array}$ & $0(0)$ & $\begin{array}{l}3.0(1.0- \\
5.0)\end{array}$ & $\begin{array}{l}1.0(0.0- \\
3.0)\end{array}$ & $<0.01$ \\
\hline $\begin{array}{l}\text { Length of stay, } \\
\text { median (IQR) }\end{array}$ & $\begin{array}{l}3.6(1.8- \\
6.9)\end{array}$ & $\begin{array}{l}5.8(2.9- \\
10.6)\end{array}$ & $\begin{array}{l}4.5(2.1- \\
8.7)\end{array}$ & $<0.01$ \\
\hline
\end{tabular}

${ }^{*} N$ ranges 2,030-2,200 because of missing values for some variables, including 2,030 for BMI

Our most striking results relate to variation in telehealth use across sites of care. Local culture and the possibility of implicit bias likely contributed to variation in telehealth adoption. Future research should explore whether differences in telehealth practice patterns are associated with patient outcomes.

Our study was conducted in a single healthcare system, potentially affecting generalizability of our findings. Furthermore, identification of telehealth visits may have been
Table 2 Adjusted Incidence Rate Ratio of Telehealth Visits During Hospitalization for COVID-19*

\begin{tabular}{|c|c|c|}
\hline & $\begin{array}{l}\text { Adjusted incidence } \\
\text { rate ratio }(95 \% \text { CI) } \\
N=1,920\end{array}$ & $P$ value \\
\hline Age & $1.00(0.99-1.00)$ & 0.58 \\
\hline Female (male as ref.) & $0.95(0.89-1.01)$ & 0.12 \\
\hline \multicolumn{3}{|l|}{ Race (White as ref.) } \\
\hline Black & $1.08(0.99-1.18)$ & 0.08 \\
\hline Asian & $0.94(0.78-1.13)$ & 0.51 \\
\hline Other & $0.88(0.80-0.98)$ & 0.02 \\
\hline Latinx & $1.17(1.05-1.30)$ & $<0.01$ \\
\hline Non-English Primary & $0.92(0.84-1.01)$ & 0.08 \\
\hline \multicolumn{3}{|l|}{ Language } \\
\hline \multirow{2}{*}{\multicolumn{3}{|c|}{ Admission source, (ED as ref.) }} \\
\hline & & \\
\hline Direct admission & $0.96(0.84-1.11)$ & 0.58 \\
\hline External transfer & $0.76(0.61-0.93)$ & 0.01 \\
\hline \multicolumn{3}{|l|}{ Payer (private as ref.) } \\
\hline Medicare & $0.96(0.89-1.04)$ & 0.33 \\
\hline Medicaid & $1.11(1.02-1.21)$ & 0.02 \\
\hline Self-pay/other & $0.62(0.48-0.79)$ & $<0.01$ \\
\hline Intensive care unit stay & $0.93(0.84-1.02)$ & 0.12 \\
\hline Mechanical ventilation & $0.91(0.82-1.01)$ & 0.08 \\
\hline Elixhauser score & $1.00(1.00-1.01)$ & 0.07 \\
\hline \multicolumn{3}{|l|}{ Month (March as ref.) } \\
\hline April & $1.12(1.03-1.22)$ & 0.01 \\
\hline May & $0.79(0.71-0.87)$ & $<0.01$ \\
\hline \multicolumn{3}{|l|}{ Hospital (D as ref.) } \\
\hline A & $0.15(0.13-0.18)$ & $<0.01$ \\
\hline B & $0.24(0.19-0.31)$ & $<0.01$ \\
\hline $\mathrm{C}$ & $2.22(2.07-2.38)$ & $<0.01$ \\
\hline
\end{tabular}

*Adjusted analysis used multiple Poisson regression controlling for variables listed in the table

susceptible to errors related to incomplete documentation or incorrect abstraction.

In conclusion, we show differences in telehealth use for hospitalized COVID-19 patients based on patient characteristics, most notably race, ethnicity, and site of care. Future research should explore variation in inpatient telehealth use and whether differences are associated with patient outcomes.

Gopi J. Astik, MD MS ${ }^{1}$

Aditi Puri, MD MS ${ }^{1}$

Frank Aguilar, $M D M P H^{1}$

Shuhan Zhou, MPP ${ }^{1}$

Kevin J. O'Leary, MD MS ${ }^{1}$

${ }^{1}$ Division of Hospital Medicine, Northwestern University Feinberg School of Medicine,

Chicago, IL, USA

Corresponding Author: Gopi J. Astik, MD MS; Division of Hospital Medicine, Northwestern University Feinberg School of Medicine, Chicago, IL, USA (e-mail: gastik@nm.org).

\section{Declarations:}

Conflict of Interest: The authors declare that they do not have a conflict of interest. 


\section{REFERENCES}

1. Kakani P, et al. Patient Characteristics Associated with Telemedicine Use at a Large Academic Health System Before and After COVID-19. J Gen Intern Med. 2021;36(4):1166-1168. https://doi.org/10.1007/s11606020-06544-0.

2. Campos-Castillo C, Anthony D. Racial and ethnic differences in selfreported telehealth use during the COVID-19 pandemic: a secondary analysis of a US survey of internet users from late March. J Am Med Inf Assoc. 2021; 28(1):119-125.
3. Pierce RP, Stevermer JJ. Disparities in use of telehealth at the onset of the COVID-19 public health emergency. J Telemed Telecare. 2020:1357633X20963893. https://doi.org/10.1177/1357633X20963893.

Publisher's Note: Springer Nature remains neutral with regard to jurisdictional claims in published maps and institutional affiliations. 